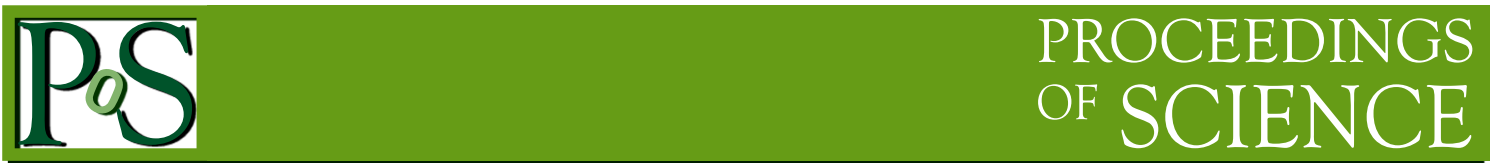

\title{
LIDAR Thomson Scattering for ITER Core Plasma Revisited
}

\section{Per Nielsen}

No affiliation

Østre Kirkevej 19, 4000 Roskilde, Denmark

E-mail: aogp@kabelmail.dk

\section{Hans Salzmann}

No affiliation

Beblostr 8, 81677 München, Germany,

E-mail: gfhmset-online.de

\section{Chris Gowers}

No affiliation

27 Cavendish Rd., Matlock DE4 3GY, UK

E-mail: chris.gowers@which.net

The authors have become aware that the development of the hitherto planned time-offlight Thomson scattering system for the ITER core plasma is not proceeding and that conventional Thomson scattering set-ups are being discussed as an alternative. In this paper, we want to point out the advantages of LIDAR and show how criticized details of the original design can be improved. We present a design of the front optics, which in neutronics terms closely resembles a layout already previously accepted. The presented design does not require Raman scattering calibration for the density measurement. Comparison with the JET Core LIDAR system and simulations at higher temperatures both show that with the new design the specified accuracy can be met with a 1-2 J laser. Current laser and detector technology is reviewed. A strategy for how to proceed is presented.

First EPs Conference on Plasma Diagnostics - $1^{\text {st }}$ ECPD

14-17 April 2015,

Villa Mondragone, Frascati (Rome) Italy 
The 17 years old design of the LIDAR TS system for the core plasma [1] is reviewed and optimized taking into account the improvements of the relevant technologies. One of the major improvements consists in a change of the optical layout whereby the need for absolute calibration by Raman scattering is avoided. The JET experience and numerical simulations show that the diagnostic is feasible with present day technologies.

\section{Review of Measurement Specifications}

The specifications for the Core Thomson Scattering System have undergone a couple of changes. The spatial resolution is now $7 \mathrm{~cm}$ and the electron temperature should be measured in the range $500 \mathrm{eV}$ to $40 \mathrm{keV}$ for densities $3^{*} 10^{19} \mathrm{~m}^{-3}$ to $3 * 10^{20} \mathrm{~m}^{-3}$.

The revised numbers were derived by the ITPA Topical Group on Diagnostics [2]. In the report, it is further stated about the central plasma with the highest temperatures and the main burning region: "The spatial resolution required is $<10 \mathrm{~cm}(\sim \mathrm{a} / 30)$ and in general a modest time resolution $(10 \mathrm{~Hz})$ is needed."

\subsection{Key Properties of LIDAR Thomson Scattering}

There is a number of attributes the LIDAR principle offers in comparison with a conventional TS system where many collection optic lines of sight have to be crossed (and maintained crossed) with the laser beam path.

Attributes special to LIDAR on ITER are: 1) Only a single penetration of the blanket , common for both the laser beam and the collected backscattered light. 2) The optical collection eptical system is relatively simple, consisting of two mirrors and no refractive elements. 3) Alignment of the collection optics to the laser beam is easily verified, even during operation. 4) The beam dump is not required as a means of stray laser light reduction. 5) Only one set of spectral channels is required for all points across the profile, simplifying calibration and reducing cost. 6) No need for Raman calibration.

These attributes are major advantages of the LIDAR system over a conventional TS approach.

\section{Lasers and Detectors}

Fast, large sensitive area photodetectors together with a laser emitting 300 ps pulses are needed to enable the specified spatial resolution. Only microchannelplate photomultipliers (MCP PMTs) with photocathodes sensitive in the Visible and NIR offer both properties. Thus a laser with emission in the NIR is required, to ensure that the decisive blue wings of the Thomson spectra overlap with the sensitive range of the MCP PMT photocathodes.

The Ti:Sapphire laser at a wavelength of $820 \mathrm{~nm}$ is a good choice, because it allows the measurements to be made with presently available detectors. For output energies of a few Joules

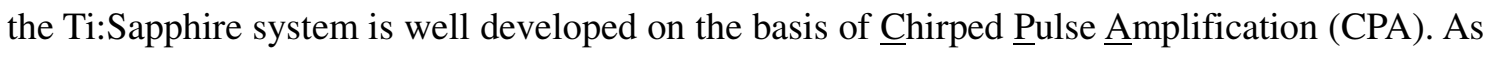
an example, in the Varennes ALLS laser system chirped pulses of 250 ps and 350 ps duration are amplified up to $5 \mathrm{~J}$ and $7.5 \mathrm{~J}$, respectively, at $10 \mathrm{~Hz}$ repetition rate [3,4]. The system is based on commercial products. Since these power levels can be tolerated in the amplifier chain, a laser for the envisaged LIDAR diagnostic could be made without using CPA techniques, simply by amplifying narrow-bandwidth seed pulses of 300 ps duration. 
Concern has been stated about the possible use of lasers with a broad gain spectrum, especially Ti:Sapphire, because of the presence of collinear Amplified Spontaneous Emission (ASE). This concern is directly linked to the "beam dump problem", where the anxiety was that in case of an imperfect beam dump the backreflected precursor ASE radiation could drown the TS signal. Reflected precursor radiation within a time interval $26.6-0.0 \mathrm{~ns}$ before the laser pulse indeed overlays the TS measurements. However, a simple estimate does not substantiate these fears: We compare the number of collected Thomson scattered photons, scattered into the total spectrum within the time interval of a measurement (300 ps) at the lowest specified density $\left(3 * 10^{19} \mathrm{~m}^{-3}\right)$, with the maximum possible number of collected ASE photons in the same time interval. We assume, as worst case situation, that $100 \%$ of these photons are collected, which corresponds to the beam dump being a perfect retroreflector.

The ASE data are taken from the ALLS laser [4]. For this system the precursor-torecompressed-laser-pulse contrast ratio is given over a rather long period in front of the laser pulse. For times $>13 \mathrm{~ns}$ in front of the laser pulse, corresponding to measurements at $\mathrm{R}>6 \mathrm{~m}$, one can extrapolate from Fig. 5 of ref. 4 values of about $10^{-16} \times 5.4 \mathrm{~J} / 25 \mathrm{fs} \approx 22 \mathrm{~mW}$, that is $\sim 10^{-}$

12 times the power of the uncompressed pulse. These are precursor power values for a laser chain producing $7.5 \mathrm{~J} / 350 \mathrm{ps}$ pulses.

In addition to the ASE discrimination used in this laser by means of the usual methods i.e. Pockels cell shutters, high energy seed pulse injection, saturable absorbers and spatial filtering, spectral discrimination can be used to further reduce the precursor power. Contrary to a CPA system this will be possible for a LIDAR laser because here the spectral bandwidth of the amplified pulse is much smaller than the bandwidth of the ASE.

The fraction of laser pulse photons scattered into the whole Thomson spectrum, into the solid angle $\mathrm{d} \Omega$ envisioned for the plasma centre, and during a sampling period corresponding to a spatial resolution of $\Delta \mathrm{L}=5 \mathrm{~cm}$, is given by $\mathrm{F}_{\mathrm{Th}}=\sigma_{\mathrm{Th}} * \mathrm{n}_{\mathrm{e}} * \Delta \mathrm{L} * \mathrm{~d} \Omega$, amounting to $\sim 5 * 10^{-14}$ for the lowest electron density specified.

Please note, that due to the finite spectral width of the ASE, the precursor radiation even without spectral narrowing overlaps with the decisive parts of the TS spectrum only at the low temperature plasma edge. Here the reflected precursor radiation $26 \mathrm{~ns}$ in front of the pulse, which again is weaker by orders of magnitude, coincides temporally with the Thomson scattered signal. At the plasma center, where the TS spectrum is much broader, the stronger ASE radiation $13 \mathrm{~ns}$ in front of the laser pulse does not overlap the important spectral channels. Hence the collected ASE will not interfere with the measurements.

To summarize, an $820 \mathrm{~nm}, 300 \mathrm{ps,} 2 \mathrm{~J}$ Ti:Sapphire laser seems feasible and well suited for LIDAR TS. However, the attainable repetition rate of about $10 \mathrm{~Hz}$ does not match the specification of $100 \mathrm{~Hz}$. Higher repetition rates seem difficult to realise with this type of laser

To realise higher repetition rates, diode pumped Yb:YAG lasers at $1030 \mathrm{~nm}$ are promising. Commercial $200 \mathrm{~mJ} / 1 \mathrm{ps} / 100 \mathrm{~Hz}$ systems are presently available and according to the producer a $2 \mathrm{~J}$ laser should be possible [5]. The use of this laser type however requires MCP PMTs with extended sensitivity in the NIR. Otherwise the low electron temperatures cannot be measured without using an additional, shorter wavelength laser. Such NIR photocathodes with $\mathrm{QE}=18 \%$ @ $960 \mathrm{~nm}$ have been demonstrated [6], but are not on offer in MCP PMTs at present.

With respect to all other properties of the MCP PMTs there has been tremendous progress recently. The response time has been shortened considerably using smaller pore size MCPs: 
Gateable, proximity focused MCP PMTs with a V-stack of two $10 \mathrm{~mm}$ dia. MCPs offering a response time of $150 \mathrm{ps} \mathrm{FWHM} \mathrm{[7]} \mathrm{are} \mathrm{available} \mathrm{as} \mathrm{well} \mathrm{as} \mathrm{larger} \mathrm{sensitive} \mathrm{area} \mathrm{(40} \mathrm{mm} \mathrm{dia.)}$ devices with 225 ps FWHM, gateable too [8]. The lifetime of the MCP PMTs of several producers has been increased by a factor of about 50 by using Atomic Layer Deposition (ALD) for coating the inside of the MCP pores, e.g. [9]. The lifetime now corresponds to one year of continuous operation at $100 \mathrm{~Hz}$ with complete charge depletion at each measurement.

\section{Front end Optics}

In this paper we only discuss the front part of the collection optics on ITER which will be located in a labyrinth in the port plug. The optical elements in this area are inaccessible over long periods of time. The elements inside the port plug are also exposed to a high flux of neutrons and to particle depositions. Refractive optics therefore cannot be considered, apart from the entrance/exit windows of the laser beam and the collected signal. The optical design aims to overcome the complex vignetting problem of the JET system, specifically to avoid the need for Raman scattering calibration.

The additional optics required to transport the signal to the spectrometer detectors is designed as a simple relay system consisting of a number of mirrors and lenses.

The collection optics at JET consist of 6 windows and 6 spherical mirrors equally spaced around the central axis of the collection system. The observation cones defined by these two groups of elements cross the midplane near the plasma centre. In addition the scattered light has to pass through a penetration in the Torus Hall ceiling. All these elements contribute to a complicated "vignetting curve". The solid angle of collection from the different positions along the plasma chord is determined through Raman scattering.

The LIDAR TS design for ITER however, makes Raman scattering unnecessary. To achieve

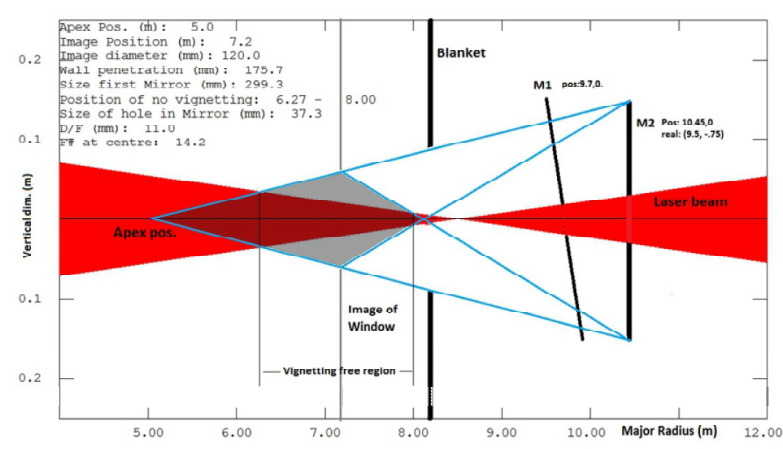

Figure 1: This graph illustrates a solution where we have chosen $\mathrm{D} / \mathrm{F}=11$ (étendue $=75 \mathrm{~mm}^{2} \mathrm{sr}$ ) and M2 located at $10.45 \mathrm{~m}$ with a diameter of $300 \mathrm{~mm}$. The slanted line at $9.7 \mathrm{~m}$ illustrates that the collected light is deflected at this location by the slightly oversized M1. A small hole in this mirror allows the laser beam to enter. this, we make sure that that the solid angle of collection of at least the outer half of the plasma is defined by two apertures only. In addition we have to ensure that all the light propagating between these two apertures reaches the detector. The design is further constrained by the fact that the étendue of the collected light beam which the detectors can accept, is limited.

To avoid neutron damage the front collection optics consists of metallic mirrors only. The mirrors have to be located inside a labyrinth in order to reduce the neutron flux to the outside of the machine. Due to reflection losses, it is further desirable to minimise the number of mirrors. The simplest solution consists of two mirrors in a dogleg configuration. At least one of the mirrors has to be imaging, imaging a location in the plasma onto the exit window. A design of a similar dogleg layout has been assessed with respect to neutron flux attenuation [10]. That design was found to fulfil the neutron screening requirements. 
We propose placing a flat mirror M1 at $\mathrm{R}=9.7 \mathrm{~m}$ (Fig. 1). This mirror deflects the signal inside the port plug onto the imaging mirror M2, $0.75 \mathrm{~m}$ away. In Fig. 1, we ignore this deflection as it plays no role in imaging terms. The laser beam enters through a hole in the flat mirror M1. The size of this hole depends on the position of the focal point, here placed at $R=8.5 \mathrm{~m}$. To avoid damage to the inner wall we have chosen to expand the beam to a diameter of $140 \mathrm{~mm}$ at the inner wall (IW). We note that a beam dump for suppression of stray light is not required.

The figure illustrates a solution that leaves the outer half of the plasma free of vignetting. Whenever the TS scattering volume is completely inside the resulting double cone

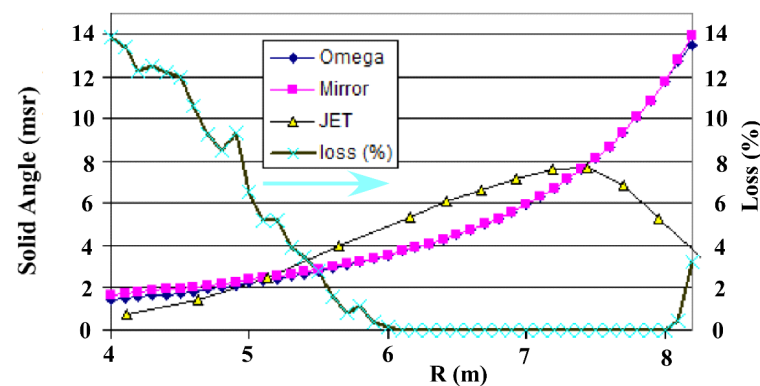

Figure 2: The curve "Omega" shows the solid angle of collection for an ITER design with a central F/15 and no vignetting of the outer half of the plasma. The "Mirror" curve shows the value you would have if the first mirror was unvignetted. Also shown for comparison is the JET vignetting curve, suitably scaled in R. (marked in Fig. 1), the solid angle of collection is given by the size of M2 and the distance to the scattering volume. We define the throughput of the system by the diameter (D) of the detector and the Fnumber $(\mathrm{F})$ of its collection cone. D/F must be maintained by the relay optics and the front optics. We have chosen as an example a value of $\mathrm{D} / \mathrm{F}=11 \mathrm{~mm}$. This value of $\mathrm{D} / \mathrm{F}$ allows the use of current JET detectors.

The presence of a laser focal point eliminates the possibility of Raman scattering because of potential breakdown of the gas. Fortunately, we do not need the Raman calibration when the outer half is free of vignetting.

Using ray tracing we calculate the complete vignetting curve. In Fig. 2. we plot the solid angle that would be valid if only M2 were the limit, together with the result from ray tracing. There is, as expected, no difference between the two vignetting curves over the range $6 \mathrm{~m}<\mathrm{R}<$ $8 \mathrm{~m}$. In addition, we see that the difference is below $5 \%$ all the way down to $\mathrm{R}=5 \mathrm{~m}$. For comparison we also show the measured Raman calibration at JET.

\section{The expected performance}

For discharges with similar parameters, the expected performance of the ITER LIDAR system can be inferred directly from the JET experiences. The background plasma light level will be obviously twice as large for ITER at the same étendue, due to the larger size. If we stay with the value of $\mathrm{D} / \mathrm{F}=11 \mathrm{~mm}$, then plasma radiation will be a factor four higher. Given the low background level observed at JET this plays no significant role. The total transmission realised in this new design is very similar to the original JET setup. We can therefore conclude that plasmas in the same range of temperatures and densities encountered at JET can be measured at ITER with a laser energy in the range $1-2$ Joule.

\subsection{Our Simulations}

In the simulation we choose a 1 Joule Ti:Sapphire laser operating at $820 \mathrm{~nm}$ and Ga:As or Ga:AsP PMTs with quantum efficiencies better than $30 \%$ over the spectral range of $400-800$ $\mathrm{nm}$. We assume a total transmission of $10 \%$, vignetting as given above and standard 
Bremsstrahlung level.

For temperatures up to $20 \mathrm{keV}$ we find that the specified measurement accuracy is met with this set of parameters. This is in agreement with our comparison to the actual performance at JET.

At the highest temperatures $(\sim 40 \mathrm{keV})$, we find that both density and temperature profiles are reasonably recovered, Fig 3(a), but the accuracy even over the outer half is not within the
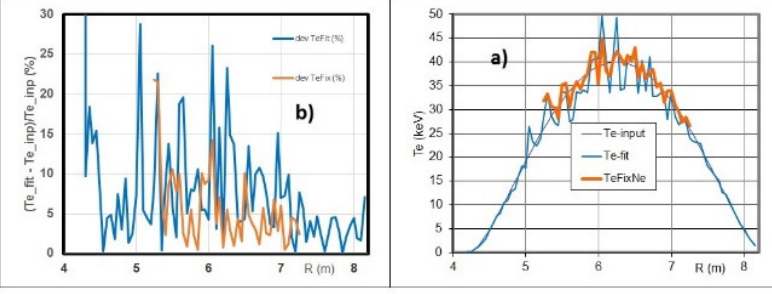

Figure 3: a) Example of fitted profiles in the simulation programme. b) shows the differences in deviation of the fit from the input profile data for the two fits. specifications, Fig. 3(b). shows the deviation relative to the input profile.

Notice, however the much better fit towards the outside, even when the density is lower than $3.10^{19} \mathrm{~m}^{-3}$. This is a result of the increasing solid angle towards the outside.

Using the expectation values at the centre we can calculate the standard deviations. For an electron temperature of $\mathrm{Te}=40 \mathrm{keV}$ and density of $3.10^{19} \mathrm{~m}^{-3}$ we calculate a standard deviation of $15 \%$ for the temperature and $8.5 \%$ for the density. Additionally the cross correlation between temperature and density inferred from the standard regression fit is 0.45 . At $20 \mathrm{keV}$ the standard deviation for Te is $5.2 \%$ and for density $2.4 \%$, while the cross correlation is reduced to 0.013 .

Fig. 4 clearly illustrates this problem. If we plot the $68 \%$ confidence level at the two temperatures we see that at $20 \mathrm{keV}$ the small cross correlation results in almost the same

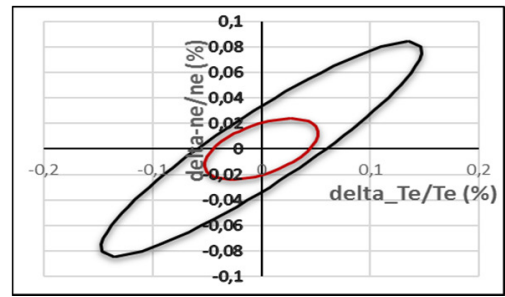

Figure 4: Contours of $\Delta \chi^{2}=1$ for $\mathrm{Te}=$ $20 \mathrm{keV}$ and $40 \mathrm{keV}$. uncertainty for the extremes of the curves as the values at the intersections of the axes. In the $40 \mathrm{keV}$ case the difference between the standard deviation and the crossing points of the axes is a factor three for the temperature and greater than two for the density. This observation leads to an alternative way of fitting the data for the high temperature region. If we knew the density, then the standard deviation of temperature would be reduced to $5 \%$. Even allowing $2 \%$ error of the density the accuracy in temperature is within $10 \%$.

We expect that such high temperatures are associated with H-mode plasmas in which case the density is constant over a wide region around the centre. If we therefore determine the central density as the average over such a region then we overcome the cross correlation problem. Using this averaged density we can make a new fit with a reduced number of free variables. This brings the fitted temperature much closer to the input profile data and the accuracy is now within the specifications. This result is also shown in Fig. 3.

It should be noted that this simulation was run at the worst imaginable conditions, low density, 1 Joule laser energy, and total optical transmission of $10 \%$, the latter being less than half the value we have estimated is possible. Increasing any of these numbers will clearly give an even better performance.

\section{Proposed Strategy}

At the beginning of ITER operation, a working TS system must be ready. This requires that all the inaccessible front components are in place. The layout of this part of the proposed optics does 
not depend on the choice of laser and detectors and thus the detailed design could be started as soon as approved by final neutron transport calculations.

Comparison with the JET LIDAR system shows that operation of such a system on ITER is possible at least up to temperatures of $15 \mathrm{keV}$. Considering the range of high spectral sensitivity of the presently available detectors we deem a $2 \mathrm{~J}, 300 \mathrm{ps}, 10 \mathrm{~Hz}$ Ti:Sapphire laser operating at $820 \mathrm{~nm}$ to be the optimum laser to start with. The available detectors allow the measurement of electron temperatures starting from the specified lower limit up to high temperatures without any gap requiring the use of an additional laser. Even a temperature of $40 \mathrm{keV}$ can be determined at low density within the specified error bars

\section{References}

[1] ITER Internal Paper WBS 5.5.C.01 (1997).

[2] A.J.H. Donné et al., Progress in ITER Physics Basis, Chapter 7, Nucl. Fusion 47, 337 (2007).

[3] S. Fourmaux et al., Laser Pulse Contrast Ratio Cleaning in 100 TW Scale Ti: Sapphire Laser Systems. INTECH Open Access Publisher, 2011.

[4] S. Fourmaux et al., Pedestal cleaning for high laser pulse contrast ratio with a $100 \mathrm{TW}$ class laser system, OPTICS EXPRESS 19 (9) 8486-8497 (2011).

[5] TRUMPF Scientific Lasers.

[6] A. Smith et al., Transmission Mode Photocathodes Covering the Spectral Range, $3^{\text {rd }}$ Beaune Conference on New Developments in Photodetection, June 17-21, 2002.

[7] J.R. Howorth et al., Ultra-Fast Photomultipliers with High Dynamic Range, HSSPP Conference September 2006.

[8] J.S. Milnes et al., Improved time response for large area microchannel plate photomultiplier tubes in fusion diagnostics, RSI 85 (11), 11E601 - 11E601-3 (2014).

[9] T. M. Conneely et al., Extended lifetime MCP-PMTs: Characterisation and lifetime measurements of AL coated microchannel plates, in a sealed photomultiplier tube, Nucl. Instruments and Methods in Physics Research A 732, 388-391 (2013). 